\title{
Information disclosure and attitude towards privacy on Facebook among undergraduates: A survey at the Eastern University, Sri Lanka
}

J.Lavanya ${ }^{1}$ and S.Santharooban ${ }^{2}$

\begin{abstract}
Users of Facebook has been increasing exponentially in the last two decades, allowing millions of people to expose their profiles to the public and with no or little privacy for individuals. Facebook, in today's context has created a concern regarding privacy of its users and the consequences of information disclosure. Based on current reports regarding negative consequences of disclosure of information on social network sites, students seem to be unconcerned about the potential negative impacts of the same. The objectives of the study are to analyze the pattern of information disclosure on Facebook, examine the relationship between information disclosure and gender, determine the methods used for making friends on Facebook and its association with gender and, measure the attitudes towards privacy on social networking sites. A quantitative research approach was employed using a self-administrated questionnaire to gather responses from undergraduates of the Eastern University, Sri Lanka. The results revealed that the majority of undergraduates disclose their real name (82.3\%), hometown (63.2\%), e-mail address $(60.53 \%)$ and, education $(62.13 \%)$, and rarely share the relationship status (32\%). Education is the highest criteria which both gender use to select friends (Male-56.12\% \& Female-55.89\%). Moreover, the results revealed that females seldom share this information than males $(\mathrm{p}<0.05)$. Further, there was a tendency, to share different information with different groups of people which was statistically significant. The favourite, events, and location are associated with friends, while location and opinions are associated with
\end{abstract}

\footnotetext{
${ }^{1}$ Assistant Librarian, Eastern University, Sri Lanka. Email:

1.lavanya08@yahoo.com

(1) http://orchid.org/0000-0002-8567-8202

${ }^{2}$ Senior Assistant Librarian, Eastern University, Sri Lanka. Email: santharoobans@esn.ac.lk iD http://orchid.org/0000-0003-1984-875X
} 
the public. However, political views, and photos are appearing towards not sharing category. Relatively $41.1 \%$ of respondents were satisfied with the privacy setting of SNS. It gives evidence that privacy settings are still neglected, and it stresses the need for awareness programmes on privacy concerns on SNS for undergraduates.

Keywords: Social Networking Sites, Facebook, Information Disclosure, Privacy Settings

\section{Introduction}

Social networking sites (SNSs) have transformed communication process and foster social interaction in a virtual environment. In this digital era, these sites have penetrated throughout the world. There are plenty of social networking sites, such as Facebook, WhatsApp, Viber, Instagram, etc., that serve as a forum where people share their opinions, life events, photos, and even feelings and thus it becomes a part and partial of life for many people. Information sharing is encouraged by SNSs by giving multiple options to allow people to post their information on their wall and their friends' wall (McGuinness \& Simon, 2018). In the recent context, undergraduates use Facebook as a tool for social interaction, building their friendship network, and showcase their personal identity and even for educational purposes as well (Debatin et al., 2009). On Facebook, people get involved in various activities, such as chatting, uploading videos, photos, business flyers, and so on, and share a tremendous amount of information, such as photos, location, relationship status, education, contact details, date of birth, interests, and some private moments of their life. Such information when available in virtual space can be accessible not only for friends but also for strangers. This information can be grabbed by malicious people, and they can exploit the sensitive information for various unethical purposes compromising privacy and security that may bring serious implications (Henson et al., 2011).

In the contemporary world, the attitude on the revelation of personal information publically and accepting an unknown person as a friend increasingly becomes widespread on SNSs. Many SNS users have a positive attitude towards social media, and they believe that online communication is secured and they reveal personal information to the public without concerning privacy issues. SNS users post information with a specific 
audience in mind and leaving information open to strangers. However, online privacy is at risk when users underestimate the visibility of their profiles and fail to enforce adequate privacy measures (McGuinness \& Simon, 2018). Nowadays, information sharing in the form of videos and photographs has become popular on social networking sites among undergraduates as well (Paradise, 2012). Pempek et al. (2009) reported that although students can communicate privately with one another on Facebook, they were twice as likely to post messages on each others' walls as to send private messages to each other. In recent years, a concern specifically for students who are active users of SNSs, has been raised regarding their uploading photos on SNSs, especially when sensitive contents are available in the images (Paradise, 2012).

\section{Research Problem}

Over the past two decades, Facebook has become integral in the life of every individual, especially university undergraduates. Debatin et al. (2009) stated that students are rigorously engaged in Facebook, and their lives without Facebook are almost unbelievable. They create their accounts without any difficulties and connect not only for recreational activities, such as sharing information, video, photo, opinion, but also for academic purposes. However, when they share personal information with a larger audience, there may be a chance to become an online victim. There are many privacy breaches and identified theft cases that are increasing rapidly (Gharibi \& Shaabi, 2012; Gangopadhyay \& Dhar, 2014). Further, exploring attitude towards information disclosure, information sharing, privacy settings, and making friends on Facebook need to be investigated among students. This would help formulate strategies to get rid of a certain degree of risk. After a literature review on the privacy of SNSs, especially on Facebook, it became evident that there is a lack of research carried out on university student's privacy on Facebook in the Sri Lankan context. Therefore, this research provides significant academic information on the type of personal information disclosed in social media and their attitudes towards privacy and the relationship between information disclosure and gender. 


\section{Objectives of the Study}

The general objective of the study is to explore information disclosure and attitude towards privacy on Facebook among undergraduates of the Eastern University, Sri Lanka

The specific objectives are:

I. To analyze the pattern of information disclosure on Facebook

II. To examine the relationship between information disclosure and gender

III. To determine the methods for making friends on SNS and its association with gender

IV. To examine the attitudes towards privacy on Social Networking Sites

\section{Literature Review}

This section reviewed the literature relevant to information disclosure on SNS and attitude towards privacy concerns on SNS in the foreign and Sri Lankan context. Social media has turned into a powerful tool for communication in the information age and plays a significant role in students' lives. It serves as an enormous platform to share their innovative ideas, similar interests, relationships, etc. This study would be an eye-opener for new avenues of scholarly investigation, such as student information disclosure on SNS, sharing information, making friends, and attitude towards privacy.

The past two decades have seen a rapid development in the number of people utilizing SNSs. Gray (2017) reported that Facebook was the third highly accessed website after Google and YouTube in June, 2016. Even though SNSs give numerous benefits for people, such as keeping in touch with companions and family, privacy and security are considered fundamental issues that can endanger the users (Donath, 2007). Therefore, there is a need to initiate research on SNS and focus on privacy concern among undergraduates since students seem to be unaware of the detrimental effects caused by improper usage of social networks and information disclosure.

There were several studies, performed to explore on the information disclosure on SNS. Johani (2016) conducted a study on personal information disclosure and privacy in SNSs. The results showed that a considerable 
amount of personal information is revealed through SNSs, and a high-level of information disclosure is observed on Facebook than other SNSs. Similarly, a study conducted by McGuinness and Simon (2018) on university students reveals that the students were concerned about online privacy and cautious about information sharing and its protections in SNSs. The sharing of information in SNSs is influenced by several factors. Mostly demographic factors influence the pattern of information disclosure and privacy settings. As per Johani (2016), information disclosure in SNSs is influenced by gender, age, and education. Older people are more concerned on sharing personal information and personal privacy than youngsters (Williams et al., 2009; Zukowski \& Brown 2007).

Some literature address the positive side of sharing information on SNSs. Nuskiya (2017) conducted a study on the impact of social media among the students of South Eastern University of Sri Lanka. The result reported that students are highly engaged in Facebook, positively impacting their academic performance and improving their skills. Social media was used for various purposes, such as collecting notes, conducting research, finding jobs, finding innovative ideas, making contacts with professionals on demand, and sharing their opinions without hesitation.

There are ways to mitigate the privacy risk, coming through SNSs. Those are avoid sharing sensitive information like address and date of birth, make users more aware of the risk, maintain a privacy management system, and continuously updating privacy policies (Gharibi \& Shaabi, 2012). In addition to making awareness and limiting personal information in SNSs, Williams et al. (2009) recommend being cautious when interacting with strangers in SNSs.

Some studies assessed privacy and attitude. There are arguments that age and gender are heavily associated with an individual's attitude towards their privacy. Younger groups were more interested in using social networks frequently and having massive networks (Kezer et al., 2016). Fogel \& Nehmad (2009) reported that women perceive more risk online and have more privacy concerns than men. A study conducted among the Indian youths revealed a relationship between attitudes toward privacy settings and enactment of privacy (Gadekar \& Pant, 2015). The study further reports that 
privacy concerns and enactment of privacy settings differ between males and females, even though the majority of them are aware of the privacy setting of Facebook.

The privacy settings are not that much complicated, but only the users' negligence is the main reason for avoiding privacy settings. In a study conducted by Al-Saggaf (2016), very few felt that privacy settings are difficult while most were comfortable with that. Cranor, Guduru, \& Arjula (2006) noted that the majority of users rarely attempt to the settings despite the user-friendly interfaces of SNSs. According to them, the reason for the negligence is lack of time and fear of "messing up" the settings. Users may have a relaxed attitude towards privacy and its associated risk as pointed out by Gross \& Acquisti (2005); however, there may be a chance to be trapped by third parties like hackers, if users do not have proper knowledge of security measures. Based on the previous research findings, it was noted that there is a dearth of research conducted in Sri Lanka, especially in relation to undergraduates, hence this research would be of importance.

\section{Methodology}

This study employed a quantitative research approach and a cross-sectional time horizon. The population of this study was students from six faculties: Agriculture, Arts and Culture, Commerce and Management, Health-Care Sciences, Science and Technology of the Eastern University, Sri Lanka. Population size is four thousand six hundred and thirty four (4634) students. The sample size was calculated using Krejcie \& Morgan's (1970) formula and $10 \%$ of the sample size was added to overcome the non-responses, and thus the final sample size was 391 . To ensure the proportionate allocation of gender, stratified simple random sampling using gender as a stratum was employed for the sample selection.

The data collection tool was a self-administered questionnaire, consisting of questions on demographics, information disclosure, sharing personal information, making friends on Facebook, and attitude towards privacy on SNS. Some statements were designed to identify participants' general concerns/attitude over privacy on SNS in this survey. To identify the attitudes regarding SNS privacy-related concerns, undergraduates were requested to respond to statements using a five-point Likert scale ranging from strongly 
agree to strongly disagree. A pilot test was conducted to ensure the reliability of the study tool. Reliability was tested using the Cronbach alpha test. The average Cronbach alpha for the questionnaire is 0.8. It seems that the tool is acceptable for data collection. The entire survey was completed with a response rate of $95 \%$. Data analysis involved descriptive statistics. The Chisquare test and correspondence analysis have been performed to determine the statistical association between the variables. The significance test was done to check compatibility with $95 \%$ of the confidence interval.

Ethical clearance was taken from the Ethics Review Committee of Faculty of Health-Care Sciences, Eastern University, Sri Lanka (E/2018/76) before conducting the study. Informed consent was obtained from the participants before data collection. Anonymity and confidentiality of the information provided by the participants were ensured.

\section{Results and Discussion}

Of all the students approached, females $(72.9 \%)$ outnumbered the males $(21.9 \%)$. The study revealed that $61.4 \%$ and $38.6 \%$ of the undergraduates are from rural and urban municipalities. Moreover, more than fifty percent $(50.1 \%)$ of the respondents were from the Faculty of Arts and Culture based on proportionate sample allocation (Table 1).

\section{Table 1: Undergraduate's profile}

\begin{tabular}{ll}
\hline Faculty & Frequency \\
\hline Agriculture & $24(6.4 \%)$ \\
Arts and Culture & $188(50.1 \%)$ \\
Commerce and Management & $57(15.2 \%)$ \\
& \\
Health-care Sciences & $45(12 \%)$ \\
Science & $48(12.8 \%)$ \\
Technology & $13(3.5 \%)$ \\
\hline Total & $\mathbf{3 7 5 ( 1 0 0 \% )}$ \\
\hline
\end{tabular}

\section{Information Disclosure on Facebook}

Self-disclosure is one aspect of SNSs, including Facebook, where the users share both visual and textual information about them (Paradise, 2012). The survey could examine respondents' information disclosure pattern on SNS. The results (Table 2) demonstrate that the vast majority of users tend to 
reveal their full name, which is $82.3 \%$ of the total respondents. However, the survey found that $49.07 \%$ of users revealed their phone numbers, $41.6 \%$, and $32 \%$ of the users disclosed their address and relationship. Further, the majority of undergraduates disclosed their home town (63.2\%), education status $(62.13 \%)$, date of birth $(61.6 \%)$, and e-mail address $(60.53 \%)$.

Table 2: Information revealed on Facebook

\begin{tabular}{llll}
\hline $\begin{array}{l}\text { Information } \\
\text { revealed }\end{array}$ & $\begin{array}{l}\text { Total Frequency } \\
\mathbf{N = 3 7 5}\end{array}$ & $\begin{array}{l}\text { Male } \\
\mathbf{N = 1 0 2}\end{array}$ & $\begin{array}{l}\text { Female } \\
\mathbf{N = 2 7 3}\end{array}$ \\
\hline Real name & $308(82.3 \%)$ & $95.92 \%$ & $77.19 \%$ \\
Real photo as profile & $174(46.4 \%)$ & $86.73 \%$ & $31.18 \%$ \\
Picture & & & \\
Home town & $237(63.2 \%)$ & $87.76 \%$ & $55.13 \%$ \\
Address & $156(41.6 \%)$ & $73.47 \%$ & $30.04 \%$ \\
E-mail address & $227(60.53 \%)$ & $78.57 \%$ & $54.37 \%$ \\
Phone number & $184(49.07 \%)$ & $73.47 \%$ & $40.68 \%$ \\
Date of birth & $231(61.6 \%)$ & $82.65 \%$ & $53.61 \%$ \\
Relationship & $120(32 \%)$ & $56.12 \%$ & $23.57 \%$ \\
Education & $233(62.13 \%)$ & $78.57 \%$ & $57.03 \%$ \\
\hline
\end{tabular}

The results show that $86.73 \%$ and $31.18 \%$ of the males and females reveal their profile picture, respectively. Around $73.47 \%$ of males share their address and phone number (Table 2). This may be due to attention-seeking purposes. Gross \& Acquisti (2005) stated that there were no differences in the amount of information revealed by men and women except for phone numbers. Further, the researcher mentioned that those male users were interested in revealing their phone numbers to make their contact details accessible to all.

Only less than $50 \%$ of the females revealed information on address and phone number on SNS. This seems that females are concerned about what should and should not be revealed on social media. Revealing sensitive information on SNS, such as an address, hometown, and phone number can make users, especially undergraduates, vulnerable to blackmail, harassment, and identity theft. Therefore, users should be aware of sharing content in their profiles to prevent such issues. Social media users should refrain from posting personal information such as birthdays, interests, and locations 
visited, etc. to prevent the usage of such information for any illegal activities by malicious people (Cheung \& Lee, 2015).

\section{Sharing Information on Facebook}

The respondents were asked about types of information shared with different types of people (Table 3). More than $40 \%$ of the undergraduate reported that much of the information posted on Facebook was available to friends only excluding political views. Around $43 \%$ of the undergraduates stated that they do not want to share political views with anyone. However, less than $30 \%$ of respondents reported sharing of political views with the public. Photographs were generally restricted to friends only. When the users share their pictures, interests, events, opinions, and political views with the public, it will bring them new friendships. At the same time, it has been used to capture personal information as well. As per the survey results, $45.7 \%$ of the undergraduates preferred to share their photos with friends. Only $13.4 \%$ liked to share their photo with the public.

Table 3: Types of information shared among different groups

\begin{tabular}{lllll} 
Information & Public & Friends & $\begin{array}{l}\text { Friends of } \\
\text { friends }\end{array}$ & $\begin{array}{l}\text { Do not } \\
\text { share with } \\
\text { anyone }\end{array}$ \\
\hline My photo & $47(13.4 \%)$ & $161(45.7 \%)$ & $26(7.4 \%)$ & $118(33.5 \%)$ \\
Friends' photo & $32(9.3 \%)$ & $165(47.8 \%)$ & $52(15.1 \%)$ & $96(27.8 \%)$ \\
Family photo & $24(7 \%)$ & $155(45.4 \%)$ & $33(9.6 \%)$ & $130(38 \%)$ \\
Favorites & $76(22.5 \%)$ & $194(57.2 \%)$ & $31(9.1 \%)$ & $38(11.2 \%)$ \\
Events & $70(21 \%)$ & $179(53.5 \%)$ & $31(9.3 \%)$ & $54(16.2 \%)$ \\
Location visited & $86(25.8 \%)$ & $167(50.2 \%)$ & $35(10.5 \%)$ & $45(13.5 \%)$ \\
Political view & $86(27.9 \%)$ & $72(23.4 \%)$ & $17(5.5 \%)$ & $133(43.2 \%)$ \\
My opinions & $94(28 \%)$ & $141(42 \%)$ & $27(8 \%)$ & $74(22 \%)$ \\
\hline
\end{tabular}

To visualize this pattern, correspondence analysis was done, and the result was significant $\left(\chi^{2}=283.19, \mathrm{p}<0.0001\right)$. Two-dimensional solutions were enough to explain $96 \%$ of the variation in data. The asymmetric bi-plot (Fig. 1) clearly shows how each type of information is associated with different categories of people compared with others. This plot gives a different approach to better understand the information sharing pattern than interpreting merely by numbers and percentages. As such, mostly the political view, their photos, family photos are mostly associated with 'Do not 
share' category when compared to the favourites, events, and locations. Friends' photos are associated with friends of friends, which generally include friends as well. Favourites and events are mostly associated with friends, while locations visited, and opinions are associated with the public. The results revealed that undergraduates of the Eastern University, Sri Lanka are cautious about sharing information on SNS.

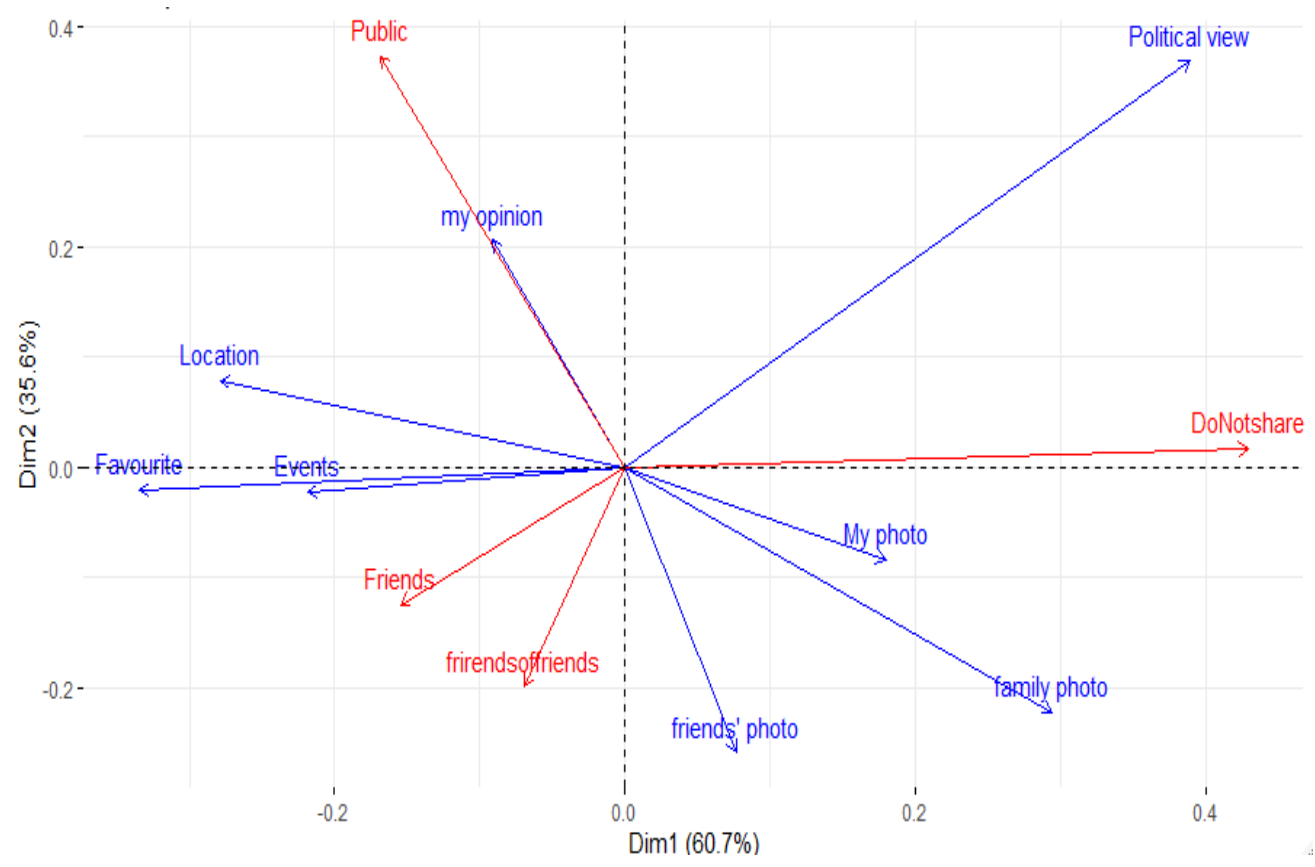

\section{Figure 1: Asymmetric bi-plot showing tendency of sharing different information among different category of people}

\section{Relationship between Information Disclosure and Gender}

The chi-square test revealed that there was a statistically significant difference $(p<0.05)$ among genders in disclosing the information. Moreover, Cramer's V test was done to indicate the strength of the association between gender and information revealed on SNS. The findings (Table 4) showed that gender, and information revealed such as real name, email, phone numbers, date of birth, and education have a significant but small association. Home town, address, and relationship have a significant but medium association with gender. However, real photos as profile pictures and gender have a significant but large association; that is; males highly reveal all kinds of 
information stated above than females. Further, females are more seldom to reveal information. Females showed a greater level of awareness of privacy protection than males (Hoy \& Milne, 2013).

\section{Table 4: Information revealed on Facebook and gender}

\begin{tabular}{llll}
\hline Information revealed & $\begin{array}{l}\text { Male } \\
\mathbf{N = 1 0 2}\end{array}$ & $\begin{array}{l}\text { Female } \\
\mathbf{N = 2 7 3}\end{array}$ & Cramer V \\
\hline Real Name & $95.92 \%$ & $77.19 \%$ & $0.22^{*}$ \\
Real photo as profile picture & $86.73 \%$ & $31.18 \%$ & $0.50^{* * *}$ \\
Home town & $87.76 \%$ & $55.13 \%$ & $0.30^{* * *}$ \\
Address & $73.47 \%$ & $30.04 \%$ & $0.39^{* *}$ \\
E-mail & $78.57 \%$ & $54.37 \%$ & $0.22^{*}$ \\
Phone number & $73.47 \%$ & $40.68 \%$ & $0.29 *$ \\
Date of birth & $82.65 \%$ & $53.61 \%$ & $0.27 *$ \\
Relationship & $56.12 \%$ & $23.57 \%$ & $0.31^{* *}$ \\
Education & $78.57 \%$ & $57.03 \%$ & $0.20^{*}$ \\
\hline
\end{tabular}

Effect size: *Small;**Medium; ***Large

Methods for making friends on Facebook and its association with gender

\section{Criteria for Making Friends}

Overall, the highest percentage of undergraduates tend to make friends based on education, followed by personal likeness. Around $56.12 \%$ and $55.89 \%$ of males and females choose their friends based on education. Besides, 55.1\% of males selected friends based on personal likeness; however, only $35.74 \%$ of females chose this option. Based on the percentage of results, females and males make friends based on a profile picture are $8.37 \%$ and $28.5 \%$, respectively. However, a small percentage of students have the habit of making friends to get someone for chatting (Male-13.27\% and Female4.18\%). It states that students give high priority to their personal preferences and likeness when compared to other reasons. It is a prominent social behaviour dominantly divulged among the undergraduates (Table 5).

The chi-square test revealed that there is a statistically significant association $(p<0.05)$ between gender and criteria for making friends on SNS especially on " based on personal likeness", "based on the profile picture", "get someone for chatting", and "earn more friends". It seems that males are highly interested in making friends on the criteria mentioned above compared to females. 
Table 5: Criteria for making friends on Facebook and Gender

\begin{tabular}{lll}
\hline \multicolumn{1}{c}{ Criteria } & \multicolumn{2}{c}{ Gender } \\
\cline { 2 - 3 } & $\begin{array}{l}\text { Male (\%) } \\
\text { N=102 }\end{array}$ & $\begin{array}{l}\text { Female (\%) } \\
\text { N=273 }\end{array}$ \\
\hline Based on education & 56.12 & 55.89 \\
Based on gender & 30.61 & 25.86 \\
Based on personal likeness & $55.10^{*}$ & 35.74 \\
Based on the profile picture & $28.57^{*}$ & 8.37 \\
Just to get someone for chatting & $13.27^{*}$ & 4.18 \\
To earn more friends & $26.53^{*}$ & 10.65 \\
\hline
\end{tabular}

*significant at an alpha of 0.01

Accepting friend request of people who never met

The responses were checked for the question "do you ever accept friend requests from people who you have never met in person"? Around $38.5 \%$ of the students stated that they would never accept requests from people they had never met, while $41.6 \%$ of students stated they sometimes accept such request (Table 6). It seems that the students do not accept random friend requests and do have privacy in mind when making decisions. Accepting a friend request from a fake profile, without thoroughly checking, leads the hacker to grab all sensitive information. This would create some serious psychological issues. Thus, accepting strangers as friends is not a healthy practice and it may lead to a certain degree of risk. Pempek et al.(2009) indicated that Facebook was heavily used for social interaction, especially with friends they know personally. The findings of Pempek et al.(2009) align with the findings of the present research.

Cross-tabulation between gender and friending people who have never met revealed that there was a significant and large association between gender and friending $\left(\chi_{(4, N=340)}^{2}=61.271, p<.001, V=0.419\right)$. Females rarely accept the request from strangers unlike males. Al-Saggaf (2016) indicated that the females were cautious in accepting friend requests, and they added only people they know and make their profiles private. It clearly states that these women have an understanding of what privacy means in their context and what kind of information they consider as private and what they need to do to maintain their privacy. 
Table 6: Accepting friend request of people who never met

\begin{tabular}{llll}
\hline Decisions & $\begin{array}{l}\text { Overall } \\
\mathbf{N = 3 7 5}\end{array}$ & $\begin{array}{l}\text { Male } \\
\mathbf{N = 1 0 2}\end{array}$ & $\begin{array}{l}\text { Female } \\
\mathbf{N = 2 7 3}\end{array}$ \\
\hline Never & $38.5 \%$ & $8.5 \%$ & $91.5 \%$ \\
Seldom & $13.7 \%$ & $14.9 \%$ & $85.1 \%$ \\
Sometime & $41.6 \%$ & $41.0 \%$ & $59.0 \%$ \\
Usually & $4.0 \%$ & $75.0 \%$ & $25.0 \%$ \\
Always & $2.3 \%$ & $62.5 \%$ & $37.5 \%$ \\
\hline
\end{tabular}

Adding Strangers into Facebook Profiles

Overall, $64.53 \%$ of the students check the profile of request senders before adding them to their Facebook profile. This seems that the students have some concern/awareness of the friending habits of other social media users. However, $11.47 \%$ of students send a message to confirm them (Table 7). It is also a good practice as the user can easily identify the intention of the friend requester at the initial stage and block their profile. This finding contradicts the findings of Johani (2016) who reported that the majority of the users of Facebook, Instagram, and Twitter were likely to accept strangers. However, the present study reveals that undergraduates nowadays are more vigilant about adding friends into SNS profiles.

When receiving a friend request, only $12.24 \%$ of male and $4.18 \%$ of female students accepted it immediately, and this difference was statistically significant in the chi-square test $\left.\chi_{(4, N=340)}^{2}=7.78, \mathrm{p}<0.05\right)$. However, except for 'accept request' there is no statistically significant difference observed in other criteria.

Table 7: Adding strangers into Facebook profiles

\begin{tabular}{llll}
\hline & $\begin{array}{l}\text { Male (\%) } \\
\text { N=102 }\end{array}$ & $\begin{array}{l}\text { Female (\%) } \\
\mathbf{N = 2 7 3}\end{array}$ & $\begin{array}{l}\text { Overall (\%) } \\
\mathbf{N = 3 7 5}\end{array}$ \\
\hline Accept request & 12.24 & 4.18 & 6.67 \\
Check profile & 71.43 & 61.98 & 64.53 \\
Check friend list & 43.88 & 41.06 & 40.53 \\
Send message & 13.27 & 11.03 & 11.47 \\
& & & \\
\hline
\end{tabular}




\section{Attitude towards Privacy Settings on Social Networking Sites}

The analysis of attitude (Table 8) indicated that $77.9 \%$ of the participants have concerns about privacy and data security while only $53.9 \%$ of the students feel the privacy of personal information is protected by SNS. But, their satisfaction with the privacy settings of SNS is around $41 \%$, while the majority are in neutral and lower levels. This shows that even though the majority have a concern about privacy and data security, they do not trust the SNS fully. Lack of awareness and negligence of privacy-related settings also affected the trust. Therefore, it necessitates user education programmes on the privacy settings of SNS. Govani \& Pashley (2005) also pointed out that even though the majority claim that they know about privacy settings, nearly half of them protect their privacy on Facebook. This may increase the risk-taking attitude among the students who use SNSs over the others (Fogel \& Nehmad, 2009).

Table 8: Attitude towards privacy settings on Social Networking Sites

\begin{tabular}{|c|c|c|c|c|c|}
\hline Statement & SA & A & $\mathbf{N}$ & D & SD \\
\hline $\begin{array}{l}\text { I have concern } \\
\text { about my privacy } \\
\text { and data security } \\
\text { while using SNS }\end{array}$ & $198(52.80 \%)$ & $\begin{array}{l}94 \\
(25.07 \%)\end{array}$ & $67(17.87 \%)$ & $\begin{array}{l}10 \\
(2.67 \%)\end{array}$ & $6(1.60 \%)$ \\
\hline $\begin{array}{l}\text { I feel that the } \\
\text { privacy of my } \\
\text { personal } \\
\text { information is } \\
\text { protected by SNS }\end{array}$ & 79 (21.07\%) & $\begin{array}{l}123 \\
(32.80 \%)\end{array}$ & $\begin{array}{l}147 \\
(39.20 \%)\end{array}$ & $\begin{array}{l}17 \\
(4.50 \%)\end{array}$ & $9(2.40 \%)$ \\
\hline $\begin{array}{l}\text { I would like to } \\
\text { post (writing } \\
\text { messages) on my } \\
\text { friends' walls }\end{array}$ & $28(7.47 \%)$ & $\begin{array}{l}79 \\
(21.07 \%)\end{array}$ & $\begin{array}{l}147 \\
(39.20 \%)\end{array}$ & $\begin{array}{l}78 \\
(20.80 \%)\end{array}$ & $43(11.47 \%)$ \\
\hline $\begin{array}{l}\text { I am satisfied } \\
\text { with the privacy } \\
\text { setting of my } \\
\text { SNS }\end{array}$ & $46(12.27 \%)$ & $\begin{array}{l}108 \\
(28.80 \%)\end{array}$ & $\begin{array}{l}181 \\
(48.27 \%)\end{array}$ & $\begin{array}{l}28 \\
(7.47 \%)\end{array}$ & $12(3.20 \%)$ \\
\hline $\begin{array}{l}\text { I feel that proper } \\
\text { use of privacy } \\
\text { setting mitigates } \\
\text { the social } \\
\text { implication } \\
\text { caused by SNS }\end{array}$ & $83(22.13 \%)$ & $\begin{array}{l}136 \\
(36.27 \%)\end{array}$ & $\begin{array}{l}131 \\
(34.93 \%)\end{array}$ & $\begin{array}{l}18 \\
(4.80 \%)\end{array}$ & $7(1.87 \%)$ \\
\hline
\end{tabular}

SA: Strongly Agree, A: Agree, N: Neutral, D: Disagree, SD: Strongly Disagree 


\section{Conclusions and Recommendations}

In the present study, the majority of users of Facebook reveal their real name, hometown, education, date of birth, and e mail. Most of the male respondents shared their profile picture, address, and phone numbers. However, only a small percentage of females revealed those information. The chi-square test revealed that there was a statistically significant difference $(\mathrm{p}<0.05)$ between genders in disclosing the information. Further, students are more cautious of what should and should not be shared with the public and accepting strangers as friends. The majority of the users have a concern about privacy and data security; however, a few are satisfied with the privacy setting of SNS. It gives evidence that privacy settings are still underutilized or neglected. To alleviate the problems raised by improper use of SNS, users should control the type of information uploaded or control access to information using privacy settings. Further, it is suggested that sensitive information / controversial statements should be shared with a particular circle of people only, and users should check their default privacy settings and read the privacy policy. There is a need to fulfil students' requirement about privacy settings as a certain percentage of respondents are satisfied with privacy settings on SNS. It is recommended that university authorities educate the undergraduates on effective utilization of privacy settings on social networking sites.

\section{References}

Al-Saggaf, Y. (2016). An exploratory study of attitudes towards privacy in social media and the threat of blackmail: The views of a group of Saudi women. Electronic Journal of Information Systems in Developing Countries, 75(7), 1-16. https://doi.org/10.1002/j.16814835.2016.tb00549.x

Cheung, C. M. K., \& Lee, Z. W. Y. (2015). Self-disclosure in social networking sites: The role of perceived cost, perceived benefits, and social influence. Internet Research, 25(2), 279-299.

Cranor, L. F., Guduru, P., \& Arjula, M. (2006). User interfaces for privacy agents. ACM Transactions on Computer-Human Interaction, 13(2), 135-178. https://doi.org/10.1145/1165734.1165735 
Debatin, B., Lovejoy, J. P., Horn, A. K., \& Hughes, B. N. (2009). Facebook and online privacy: Attitudes, behaviors, and unintended consequences. Journal of Computer-Mediated Communication, 15(1), 83-108. https://doi.org/10.1111/j.1083-6101.2009.01494.x

Donath, J. (2007). Signals in social supernets. Journal of Computer-Mediated Communication, 13(1), 231-251. https://doi.org/10.1111/j.10836101.2007.00394.x

Fogel, J., \& Nehmad, E. (2009). Internet social network communities: Risktaking, trust, and privacy concerns. Computers in Human Behavior, 25(1), 153-160. https://doi.org/10.1016/j.chb.2008.08.006

Gadekar, R., \& Pant, S. (2015). Exploring Facebook users' privacy knowledge, enactment, and attitude: A Study on Indian youth. International Journal of Communication Research, 5(4), 273-283.

Gangopadhyay, S., \& Dhar, D. (2014). Social networking sites and privacy issues concerning youths. Global Media Journal-Indian Edition, 5(1)

Gharibi, W., \& Shaabi, M. (2012). Cyber threats in social networking websites. International Journal of Distributed and Parallel Systems (IJDPS), 3(1), 119-126. https://doi.org/10.5121/ijdps.2012.3109

Govani, T., \& Pashley, H. (2005). Student awareness of the privacy implications when using Facebook. Unpublished Paper Presented at the "Privacy Poster Fair" at the Carnegie Mellon University School of Library and Information Science, 9, 1-17. https://lorrie.cranor.org/courses/fa05/tubzhlp.pdf

Gray, A. (2017). These are the world's most popular websites. https://www.weforum.org/agenda/2017/04/most-popular-websitesgoogle-youtube-baidu/

Gross, R., \& Acquisti, A. (2005). Information revelation and privacy in online Social networks (The Facebook case). ACM Workshop on Privacy in the Electronic Society, 71-80. https://doi.org/10.1145/1102199.1102214

Henson, B., Reyns, B. W., \& Fisher, B. S. (2011). Security in the 21st 
century: Examining the link between online social network activity, privacy, and interpersonal victimization. Criminal Justice Review, 36(3), 253-268. https://doi.org/10.1177/0734016811399421

Hoy, M., \& Milne, G. (2013). Gender differences in privacy-related measures for young adult Facebook users. Journal of Interactive Advertising, 10(2), 28-45. https://doi.org/10.1080/15252019.2010.10722168

Johani, M. A. L. (2016). Personal information disclosure and privacy in Social networking sites [Masters thesis, Auckland University of Technology]. CORE. https://core.ac.uk/download/pdf/80334091.pdf

Kezer, M., Sevi, B., Cemalcilar, Z., \& Baruh, L. (2016). Age differences in privacy attitudes, literacy, and privacy management on Facebook. Cyberpsychology: Journal of Psychosocial Research on Cyberspace, 10(1). https://doi.org/https://doi.org/10.5817/CP2016-1-2

Krejcie, R. V, \& Morgan, D. W. (1970). Determining sample size for research activities. Educational and Psychological Measurement, 30, 607-610. https://doi.org/https://doi.org/10.1177/001316447003000308

McGuinness, D., \& Simon, A. (2018). Information disclosure, privacy behaviours, and attitudes regarding employer surveillance of social networking sites. IFLA Journal, 44(3), 203-222. https://doi.org/10.1177/0340035218785389

Nuskiya, A. F. (2017). The Impact of social media among the university students: Empirical study based on the South Eastern University of Sri Lanka. Journal of Information Systems \& Information Technology (JISIT), 2(1), 16-21.

Paradise, A. (2012). Chapter 13 Picture Perfect? College students' experiences and attitudes regarding their photo-related behaviors on Facebook. In L. A. Wankel \& C. Wankel (Eds.), Misbehavior Online in Higher Education (Cutting-Edge Technologies in Higher Education) (Vol. 5, pp. 261-292). Emerald Group Publishing Limited, Bingley. https://doi.org/10.1108/S2044-9968(2012)0000005015

Pempek, T. A., Yermolayeva, Y. A., \& Calvert, S. L. (2009). College students' social networking experiences on Facebook. Journal of 
Applied Developmental Psychology, 30(3), 227-238. https://doi.org/10.1016/j.appdev.2008.12.010

Williams, K., Boyd, A., Densten, S., Chin, R., Diamond, D., \& Morgenthaler, C. (2009). Social networking privacy behaviors and risks. http://csis.pace.edu/ ctappert/srd2009/a2.pdf

Zukowski, T., \& Brown, I. (2007). Examining the influence of demographic factors on internet users' information privacy concerns. Proceedings of the 2007 Annual Research Conference of the South African Institute of Computer Scientists and Information Technologists on IT Research in Developing Countries - SAICSIT '07, 197-204. https://doi.org/10.1145/1292491.1292514 